Method The decision aids were developed in accordance with the International Patient Decision Aid Standards (IPDAS). A systematic search and evidence assessment were performed to analyse the benefits and risks of screening. The decision aids were tested in focus groups and surveys. The process also included a public commenting procedure.

Results Most users perceived the decision aids to be informative, helpful and balanced. The majority found the information on harms of screening relevant, including overdiagnosis and overtreatment. Healthcare professionals were more reluctant to provide information on harms in the decision aids, fearing it might lead to lower participation in screening. The surveys showed that the decision aid only had little impact on screening intention. The majority would participate in the screening programmes, despite the possible harms. The results of user testing concerning acceptance of the decision aids and screening intention were quite similar across the different decision aids. Larger differences occurred for example in the acceptance of certain graphical formats.

Conclusions Decision aids are a useful tool to support informed choice in an organized screening programme. They were well accepted by most users. There is further need for research on the implementation of the decision aids.

\section{BELIEFS, KNOWLEDGE, AND UTILISATION LEVELS OF EVIDENCE-BASED PRACTICE AMONG NURSES AND MIDWIVES IN THE REPUBLIC OF IRELAND}

Joanne Cleary-Holdforth. Dublin City University, Dublin 9, Ireland

10.1136/bmjebm-2019-EBMLive.31

Objectives Evidence-based practice (EBP) is an approach to healthcare that combines the use of the best available evidence, the healthcare professional's expertise, and patient preferences/values, when making healthcare decisions. Application of EBP in healthcare yields economical delivery of optimal patient outcomes, standardizes practice, enhances practitioner job satisfaction and empowerment and is therefore a particularly relevant concept in the current climate. Despite this, there is little evidence of EBP among healthcare professionals, including nurses and midwives. In the absence of EBP in healthcare, questions may arise in relation to the standard of care provided, the answers to which may not always be satisfactory or indeed defensible. It is therefore paramount that care afforded to patients is always based upon the best available evidence, in order to ensure optimal outcomes for all concerned. Integral to the success of any EBP initiative are the individual practitioners who deliver patient care and the organisations in which they work. Practitioners require relevant knowledge and skills to apply EBP to patient care delivery. This is particularly important for nurses and midwives, one of the largest groups of healthcare practitioners, and who are the key focus of this study. Furthermore, the organisational culture must be open and prepared to embrace and support EBP. In order to make EBP a reality in nursing and midwifery practice, its implementation must be tackled at organisational and individual levels within both the clinical and educational settings responsible for nurse and midwifery education. The current EBP beliefs and utilisation levels among nurses and midwives along with the organizational culture and readiness within these settings must be established in order to determine the current situation and the best way forward in the Irish context. This national study sets out to do this. Data will be collected from under-graduate nursing and midwifery students and lecturers in nine Higher Education Institutes in Ireland, along with staff nurses and midwives and nurse and midwife managers in affiliated clinical services. The findings of this study will inform the development of an implementation strategy at individual, organisational and potentially national levels to foster a sustainable culture of EBP use among nurses and midwives within the Irish healthcare system.

The objectives of this $\mathrm{PhD}$ study are;

1. To establish the beliefs, knowledge level and utilisation of evidence-based practice (EBP) of;

- lecturers who teach on undergraduate, pre-registration nursing and midwifery programmes delivered in Higher Education Institutes (HEIs) in the Republic of Ireland

- students undertaking undergraduate, pre-registration nursing or midwifery programmes delivered in these HEIs.

- clinically-based nurses and midwives working in teaching hospitals affiliated with these HEIs?

2. To determine the culture and preparedness of the HEIs and hospitals (within which these nurses and midwives, student nurses and student midwives, nurse and midwifery lecturers work, learn and teach) for EBP?

3. To establish informed starting point from which a specifically tailored EBP education programme, underpinned with specific knowledge of the needs of the nurses and midwives, as well as the challenges and opportunities present in their workplaces, can be developed and implemented where necessary.

Method A national cross-sectional survey of three cohorts of nurses/midwives (hospital-based nurses/midwives, nurse/midwifery lecturers, and student nurses/midwives) was undertaken. This quantitative study used three EBP questionnaires that explored EBP beliefs, implementation levels, and organisational culture and readiness for EBP, respectively. One open-ended question was included on the survey which invited participants to write down in their own words, what they believe EBP to be, the purpose of which was to gain insight into participants' knowledge and understanding of EBP. Power analysis determined adequate sample sizes fo the three cohorts, and these were achieved. Randomised sampling was used to select nine Higher Education Institutions (Schools of Nursing/Midwifery), and seven teaching hospital sites. Descriptive, correlational and inferential statistics were used to analyse the relationships among EBP attitudes and beliefs, knowledge level and utilization. Content analysis facilitated exploration of the participants' responses to the open question on the survey.

Results Quantitative results revealed that overall, across the three cohorts of participants, there is a positive attitude towards, and belief in the potential of EBP to produce improved patient outcomes. Beliefs in practitioners' own ability to implement EBP is not as positive. Utilisation levels of EBP are poor across all three cohorts, with clinical nurses and midwives scoring lowest in this area. Further insights that contribute to some explanation of these findings have been extrapulated from closer scrutiny of the quantitative data, but also from exploration of the qualitative data, which reveal poor knowledge and understanding of what EBP actually is, 
across all three cohorts. Organisational culture and readiness for EBP in the selected Schools of Nursing/Midwifery, and in the selected hospitals, was found to be moderate, but with a lot more to be done.

Conclusions The positive beliefs in EBP, and moderate organisational culture/readiness for EBP are encouraging findings. Nurses/midwives in academic and clinical settings are positively predisposed to EBP and believe their workplaces as moderately supportive of its implementation. However, the very low EBP implementation levels are concerning, both for nurse/midwifery education and practice going forward, and particularly for patient care and safety. Close scrutiny of the quantitative data reveals a substantial deficit in knowledge and understanding of EBP among nurses and midwives, and this is clearly corroborated by the qualitative data generated by the open question. These findings offer an informed starting point from which a specifically tailored education programme, underpinned with knowledge of the specific needs of nurses/midwives, as well as challenges and opportunities in their workplaces, can be developed and implemented with the aim of developing/improving EBP knowledge and skills to foster a culture of EBP.

\section{INTEGRATED INTERVENTIONS TO REDUCE PRESSURE ON ACUTE HOSPITALS: A SYSTEMATIC UMBRELLA REVIEW}

Martin Keane, Camille Coyle, Louise Farragher, Gerald O'Nolan, Aoife Cannon, Jean Long. Health Research Board, Dublin, Ireland

\subsection{6/bmjebm-2019-EBMLive.32}

Objectives The objectives of this review were to examine integrated health system interventions that have the potential to reduce pressure on acute hospitals. The outcomes that were included for assessing reduced pressure were unplanned admissions to hospital, readmissions, length of stay in hospital, emergency department visits, and healthcare costs.

Method An initial scoping search was conducted in order to frame the parameters of the review. Following the scoping search, an information specialist developed a targeted search strategy using $\mathrm{MeSH}$ terms and keywords for the outcomes of interest. Two databases, MEDLINE and the Cochrane Database of Systematic Reviews, were included in the search. Pairs of authors screened, quality assessed, and extracted data. Heterogeneity prevented pooled analysis of the included reviews. Instead, we extracted the findings related to health system outcomes from each review and described the effectiveness for each intervention by outcome measured and by population, as well as summarising the findings of the reviews for each intervention. We calculated the degree of overlap of primary studies in our included reviews using the corrected covered area measure.

Results This review included 36 published systematic reviews and one umbrella review. Our analysis identified seven complex integrated interventions targeting adults with chronic diseases, eight interventions focusing on medical and surgical conditions among adults, and three interventions for older people. We identified a total of 13 integrated interventions between the hospital and the community aiming to reduce pressure on acute hospitals. Seven interventions focused on people with chronic diseases. Among these, self-management demonstrated good effectiveness, and the other six were moderately effective (discharge management, chronic care model, complex interventions, multidisciplinary teams, hospital at home, and interactive telemedicine). Four interventions targeting acute conditions were moderately effective (discharge management, medication management, hospital at home, and primary care near emergency department), and four emergency department interventions had low effectiveness. Three interventions focusing on older people also had low effectiveness (discharge management, case management, and specialised multidisciplinary rehabilitation for hip fracture).

Conclusions The findings indicate that there are a number of promising interventions that reduce pressure on acute hospitals for people with chronic diseases. There are also some promising interventions that reduce pressure on acute hospitals for people with medical and surgical conditions. There are currently no promising interventions that reduce pressure on acute hospitals for older people. Integrated interventions are multi-component complex interventions, and the interrelationships between these components are rarely described in the literature. Furthermore, the delivery of interventions requires a complex chain of action, delivered in health systems that combine an array of pre-existing interventions and contextual contingencies. These intervention pathways were rarely explored in the studies we reviewed. Therefore, we were unable to identify why these interventions were promising for only some patients under certain conditions. These limitations make it very difficult to translate research on integrated interventions to reduce pressure on acute hospitals into policy and practice.

\section{THE RISK OF IMPRECISE TERMINOLOGY: INCONGRUENT RESULTS OF CLINICAL TRIALS AND INCONGRUENT RECOMMENDATIONS IN CLINICAL GUIDELINES}

${ }^{1}$ Franz Porzsolt, ${ }^{1,2}$ Felicitas Wiedemann, ${ }^{3}$ Karen Schmaling, ${ }^{4}$ Robert Kaplan. ${ }^{1}$ Institute of Clinical Economics, UIm, Germany; ${ }^{2}$ Diakonie-Klinikum, Stuttgart, Germany; ${ }^{3}$ Washington State University, VancouverWA, USA; ${ }^{4}$ Stanford University, Stanford, USA

\subsection{6/bmjebm-2019-EBMLive.33}

Objectives The aim of our study is to describe the congruence in the terminology used to describe both design and outcomes of clinical trials. Terminology affects the endorsement of commonly accepted clinical experience, and of poorly defined and undifferentiated interpretations of efficacy and effectiveness. Further, choice of terms may explain the results of our congruence analysis of clinical guidelines. A review of recommendations based on 330 scenarios from 24 international guidelines for the same scenario about treatment of malignant diseases found congruence in only $15 \%$ of the corresponding recommendations. ${ }^{1}$

Method We analysed five corresponding pairs of terms recommended in six reviews articles on the reporting the outcomes of clinical trials. ${ }^{2-7}$ These pairs were efficacy/effectiveness outcome, experimental/observational trial, explanatory/pragmatic attitude, randomized/non-randomized allocation of intervention, and analytical/descriptive trial. We also analysed four corresponding triplets of terms used in 100 published quality of life (QoL) studies. The references of these 100 QoL studies were reported previously. ${ }^{8}$ The four triplets of terms were explanatory/pragmatic/not defined attitude, randomized/nonrandomized/not defined allocation of intervention, primary/secondary/not defined endpoint, and efficacy/effectiveness/not defined outcome. References in online version.

Results None of the reviews included all six pairs of terms. The pairs explanatory/pragmatic, and randomized/non- 